LAWRENCE LIVERMORE N A T IO N A L LABORATORY
Spectroscopic Properties and Potential Energy Curves of Low-Lying Electronic States of RuC

R. Guo, K. Balasubramanian

December 29, 2003

Journal of Chemical Physics 
This document was prepared as an account of work sponsored by an agency of the United States Government. Neither the United States Government nor the University of California nor any of their employees, makes any warranty, express or implied, or assumes any legal liability or responsibility for the accuracy, completeness, or usefulness of any information, apparatus, product, or process disclosed, or represents that its use would not infringe privately owned rights. Reference herein to any specific commercial product, process, or service by trade name, trademark, manufacturer, or otherwise, does not necessarily constitute or imply its endorsement, recommendation, or favoring by the United States Government or the University of California. The views and opinions of authors expressed herein do not necessarily state or reflect those of the United States Government or the University of California, and shall not be used for advertising or product endorsement purposes. 


\title{
Spectroscopic Properties and Potential Energy Curves of Low-lying electronic States of RuC
}

\author{
Rui Guo and K. Balasubramanian ${ }^{\mathrm{a}, \mathrm{b}^{*}}$ \\ ${ }^{a}$ Center for Image Processing and Integrated computing, University of California \\ Davis, Livermore, CA 94550; ${ }^{b}$ University of California \\ Chemistry and Material Science Directorate \\ Lawrence Livermore National Laboratory \\ Livermore, California 94550
}

\begin{abstract}
The RuC molecule has been a challenging species due to the open-shell nature of $\mathrm{Ru}$ resulting in a large number of low-lying electronic states. We have carried out state-of-the-art calculations using the complete active space multi-configuration self-consistent field (CASSCF) followed by multireference configuration interaction (MRCI) methods that included up 18 million configurations, in conjunction with relativistic effects. We have computed 29 low-lying electronic states of $\mathrm{RuC}$ with different spin multiplicities and spatial symmetries with energy separations less than $38000 \mathrm{~cm}^{-1}$. We find two very closely low-lying electronic states for RuC, viz., ${ }^{1} \Sigma^{+}$and ${ }^{3} \Delta$ with the ${ }^{1} \Sigma^{+}$being stabilized at higher levels of theory. Our computed spectroscopic constants and dipole moments are in good agreement with experiment although we have reported more electronic states than those that have been observed experimentally. Our computations reveal a strongly bound $\mathrm{X}^{1} \Sigma^{+}$state with a large dipole moment and an energetically close ${ }^{3} \Delta$ state with a smaller dipole moment. Overall our computed spectroscopic constants of the excited states with energy separations less than $18000 \mathrm{~cm}^{-1}$ agree quite well with those of the corresponding observed states.
\end{abstract}

\footnotetext{
*Address correspondence at kbala@ucdavis.edu or fax:925-422-6810
} 


\section{INTRODUCTION}

Spectroscopic studies of transition metal carbides, in general and ruthenium carbide (RuC) in particular, have been the focus of several experimental and theoretical studies for over four decades. ${ }^{1-16}$ The nature of transition metal-carbon bonds and their low-lying states could provide considerable insight and facilitate further progresses in various related fields such as heterogeneous catalysis and organometallic chemistry. Among transition metal carbides, RuC is particularly interesting due to the open-shell character of $\mathrm{Ru}$, which yields a large number of low-lying electronic states of different spin multiplicities and spatial symmetries. Moreover, $\mathrm{RuC}$ has been one of the most studied of transition metal carbides with spectroscopic studies as early as $1970 \mathrm{~s}^{7,8} \mathrm{~A}$ particular challenge associated with Ru-containing species is that as electron correlation effects are included to higher order the nature and relative ordering of electronic states change. Consequently, RuC presents considerable challenge for theoretical computations.

There have been a number of gas-phase spectroscopic studies on RuC. Scullman and Thelin $^{7}$ have carried out an early spectroscopic study that yielded the first emission spectra in a King furnace in the $6000-8700 \AA$ range. These authors observed 46 bands that were grouped into 8 sub-systems. However, due to the complexity of the spectrum, the bands could not be assigned to the low-lying electronic states of RuC. In a subsequent study, Scullman and $\mathrm{Thelin}^{8}$ recorded the emission spectra of RuC in the range of $4100-4800 \AA$, which yielded eight more new spectral bands that could be grouped into 3 sub-systems. As noted in a more recent work by Morse and 
coworkers $^{13}$, the main difficulty in these earlier experiments is due to high temperatures $\left(\sim 3000^{\circ} \mathrm{C}\right)$ used in the preparation of the sample, which not only led to contaminations from other species like $\mathrm{C}_{2}$ and $\mathrm{CN}$, but also less population of lower rotational levels. The bond energy of RuC was measured many years ago to be about $6.5 \mathrm{eV}$ by McIntyre et al. ${ }^{9}$ and Gingerich ${ }^{10}$ using the third law method.

Langenberg et al. ${ }^{12}$ have carried out a relatively recent resonant state-of-the-art two-photon ionization (R2PI) spectroscopic study of RuC. This was followed by a dispersed fluorescence spectroscopic investigation by DaBell et al. in $2001 .^{13}$ With the advent of time-of-flight mass spectrometry and supersonically cooled molecular beam technique, Langenberg et al. ${ }^{12}$ not only obtained forty-nine bands in their experiments, but they rotationally resolved twenty-nine of those. Analysis of these bands enabled Langenberg et al. to group them into 9 subsystems, originating from three low-lying electronic states namely, $\mathrm{X}^{1} \Sigma^{+},{ }^{3} \Delta_{3}$ and ${ }^{3} \Delta_{2}$. Of the three components of the ${ }^{3} \Delta$ state, the energy difference between the ${ }^{1} \Sigma^{+}$and ${ }^{3} \Delta_{3}$ states was found to be only $75.953 \mathrm{~cm}^{-1}$, and that between the ${ }^{1} \Sigma^{+}$and ${ }^{3} \Delta_{2}$ states was observed to be $850 \mathrm{~cm}^{-1}$. The close proximity of these electronic states makes even a qualitative theoretical treatment of these states very challenging. Overall Langenberg et al. ${ }^{12}$ have reported the spectroscopic constants of 13 states (including spin-orbit coupling) for RuC. In the subsequent study DaBell et al. have added the spectroscopic constants of the ${ }^{1} \Delta_{2}$ state of RuC. Permanent electric dipole moments of two electronic states of RuC have been reported by Steimle et al. ${ }^{15}$ using the Stark shift measurements of high-resolution laser induced fluorescence spectra. 
There are very few theoretical investigations on the electronic states of RuC. Shim et al. ${ }^{11}$ have calculated the spectroscopic constants and dissociation energies for 28 electronic states of RuC using all-electron Hartree-Fock and valence configuration interaction methods. The ground state they found for $\mathrm{RuC}$ was a ${ }^{3} \Delta$ state, with a ${ }^{1} \Sigma^{+}$ state lying higher at $0.53 \mathrm{eV}$. Based on their calculations, they have assigned the emission bands observed by Scullman and Thelin. ${ }^{7,8}$ However, the ${ }^{1} \Sigma^{+}$state was assigned as the experimental ground state by Langenberg et al., in contrast to the HF/valence CI prediction of Shim et al. ${ }^{11}$ In a more recent, study Shim and Gingerich $^{14}$ have carried out all-electron calculations for the three lowest electronic states of ruthenium carbide, namely, ${ }^{1} \Sigma^{+},{ }^{3} \Delta$ and ${ }^{1} \Delta$, at the CASSCF and MRCI levels that included up to 1.3-2.9 million configurations. Relativistic effects were included in their calculations through perturbation methods. Their recent results were restricted to three low-lying states (without spin-orbit) and agree quite well with the experiment in that the ${ }^{1} \Sigma^{+}$state found to be the ground state of RuC, and the ${ }^{3} \Delta_{3}$ state lying slightly higher at about $45 \mathrm{~cm}^{-1}$. The ${ }^{1} \Delta_{2}$ state, though not observed experimentally at that time, was predicted to lie at about $6204 \mathrm{~cm}^{-1}$ above the ground state. This result was in general accord with the subsequent experimental value of $5679 \mathrm{~cm}^{-1}$ reported by DaBell et al. ${ }^{13}$ in 2001.

As seen from the above survey of experimental and theoretical studies on RuC, there is continuing interest on the low-lying states of RuC. While the most recent study of RuC employed the CASSCF/MRCI method, it was restricted to only 3 low-lying states and also to 1.3-2.9 million configurations. In our present study we 
have carried out relativistic CASSCF/MRSDCI computations that included up to 18 million configurations on 30 low-lying electronic states of RuC. We have also computed the entire potential energy curves of these states up to the dissociation limits. Thus the current computations are at a more ambitious level and include more electronic states than those studied before.

\section{METHOD OF COMPUTATION}

We start with the CASSCF method of calculations to generate the orbitals for higher-order MRSDCI computations. We have also employed the first-order configuration interaction (FOCI) method to generate the potential energy surfaces and to optimize the geometries. The CASSCF method included a full CI space of configurations obtained by distributing all valence electrons of $\mathrm{RuC}$ among the $\mathrm{Ru} 4 \mathrm{~d}$, $5 \mathrm{~s}, \mathrm{C} 2 \mathrm{~s}$ and $2 \mathrm{p}$ orbitals in all possible ways. The FOCI method included all of the configurations included in the CASSCF and all those configurations obtained by distributing one electron in the external space and remaining electrons in the internal space in all possible ways. The FOCI includes a good part of dynamic electron correlation effects, although it does not include two electron excitations into the external space, and is thus less complete than the MRSDCI method in treating dynamical electron correlation effects. Consequently, some of the lower-lying states were considered further at the first-order + multireference configuration interaction (FO+MRCI) level so as to provide a better description of those electronic states. In these FO+MRCI optimizations, all configurations in the FOCI and double excitations 
from all CASSCF configurations with absolute coefficients higher than 0.01-0.07 were included. The lower threshold of 0.01 was used to study the relative ordering of the ${ }^{1} \Sigma^{+}$and ${ }^{3} \Delta$ states. We also invoked the Davison correction technique ${ }^{17}$ (noted as MRSDCI $+Q$ ), which takes into consideration the effect of unlinked quadruple clusters. The optimizations at the FOCI and FO+MRCI levels were carried out point-wise. Then a numerical fitting procedure was employed to fit all the energy points to the potential curves from which various spectroscopic constants were then deduced. All of the calculations were carried out in the $C_{2 v}$ symmetry with the RuC molecular axis lying along z-axis of the coordinate system. Mulliken population analysis was carried out for the lower-lying electronic states of $\mathrm{RuC}$ to provide insight into the nature of bonding in $\mathrm{RuC}$.

Relativistic effective core potentials (RECPs) for ruthenium generated by LaJohn et al. ${ }^{18}$ were employed for Ru. As in the case of our previous study of $\mathrm{Ru}_{3}{ }^{19}$ the RECPs that retained the $4 s^{2} 4 p^{6}$ semicore orbitals of ruthenium as well as the outer $4 d^{7} 5 s^{1}$ shells were used for RuC. The corresponding optimized Gaussian basis sets $(5 s 5 p 4 d)$ for ruthenium, as given in Ref. 18 , were first contracted to $5 s 3 p 3 d$ and then augmented by adding one set of $f$ functions to yield $(5 s 3 p 3 d 1 f)$. The exponent of the $f$ functions was chosen to be 0.45 as in Ref. 19. However as noted below, much larger basis sets on $\mathrm{Ru}$ and $\mathrm{C}$ were also used to study the two lowest states. For carbon the RECPs that retained the outer $2 s^{2} 2 p^{2}$ shells were taken from Pacios and Christiansen. ${ }^{20}$ The optimized $(4 s 4 p)$ Gaussian basis sets for carbon were first contracted to $3 s 3 p$ and then augmented with one set of $d$ functions with exponent 0.75 
according to Dunning and Hay. ${ }^{21}$ These basis sets were used in our CASSCF calculations, FOCI and FO+MRCI optimizations. In general our FOCI calculations included near 551,000 configuration spin functions (CSFs), while our FO+MRCI calculations included up to 1.5 million CSFs.

In order to gauge the accuracy our results and to refine the relative ordering of the ${ }^{1} \Sigma^{+}$and ${ }^{3} \Delta$ states, which are clearly sensitive to the level of theory, above mentioned basis sets were enlarged by augmenting with one $p$, one $d$ and one more set of $4 f$ functions for ruthenium and one $s$, one $p$ and one more set of $3 d$ functions for carbon. This leads to a $(5 s 4 p 4 d 2 f)$ basis set for ruthenium and a $(4 s 4 p 2 d)$ basis set for carbon. These basis sets should be comparable to those used in the all-electron calculations by Shim and Gingerich. ${ }^{14}$ We employed the MRSDCI method of calculations for the lowest-lying electronic states of $\mathrm{RuC}$ and these calculations included up to 18 million CSFs. In our CASSCF calculations, the active space was chosen as the $4 d$ and $5 s$ orbitals of ruthenium along with the $2 s$ and $2 p$ orbitals of carbon, which correlate into 10 orbitals that span five $a_{1}$, two $b_{2}$, two $b_{1}$, and one $a_{2}$ orbitals. Twelve valence electrons of $\mathrm{RuC}$ were distributed among these active orbitals in all possible ways at the CASSCF level. The $4 s^{2} 4 p^{6}$ shells of ruthenium correlate into two $a_{1}$, one $b_{2}$ and one $b_{1}$ orbitals in the $C_{2 v}$ symmetry. These orbitals were allowed to relax in our calculations but excitations from these orbitals were not allowed.

Although the atomic structure of carbon atom is relatively simple with ${ }^{3} \mathrm{P}$ as its ground electronic state with the first excited ${ }^{1} \mathrm{D}$ state at about $1.26 \mathrm{eV}$ higher, 
electronic states of atomic ruthenium are much more complicate. The lowest electronic state of $\mathrm{Ru}$ is ${ }^{5} \mathrm{~F}$, but the first excited state, namely ${ }^{3} \mathrm{~F}\left(4 d^{7} 5 s^{1}\right)$ state lies at about $0.78 \mathrm{eV}$ higher, and it is closely followed by ${ }^{5} \mathrm{D},{ }^{5} \mathrm{P}$ and another ${ }^{3} \mathrm{~F}\left(4 d^{8}\right)$ states. $^{22}$ These combinations of atomic $\mathrm{Ru}$ and $\mathrm{C}$ in their respective low-lying electronic states result in singlet, triplet, quintet and heptet electronic states of $\mathrm{RuC}$ and $\Sigma^{+}, \Sigma^{-}, \Pi, \Delta, \Phi$ and $\Gamma$ symmetries. It is obvious that even if we consider the carbon atom only in its ground state, there will still be a plethora of electronic states generated from the low-lying atomic $\mathrm{Ru}$ electronic states. On the other hand, lowest singlet states of RuC can be derived only through the combination of $\mathrm{Ru}{ }^{3} \mathrm{~F}$ and $\mathrm{C}{ }^{3} \mathrm{P}$ atomic states. Considering these complexities of $\mathrm{RuC}$, multiple roots for every possible multiplicity were considered for each symmetry in the $C_{2 v}$ group at the FOCI level to facilitate a thorough exploration of the complicated manifold of electronic states. The spin-orbit parameter $^{12}$ for $\mathrm{Ru}$ is about $1038 \mathrm{~cm}^{-1}$, and thus although this number is large in spectroscopic terms, it is small in electronic structure terms as the mixing of the orbitals of Ru with carbon will keep the spin-orbit splitting lower than this number for many states. We shall discuss this aspect in more detains in the next section.

Most of our calculations were carried out using a modified version of ALCHEMY 2002, ${ }^{23-25}$ part of the CASSCF calculations were completed with GAMESS. $^{26}$ 


\section{RESULTS AND DISCUSSIONS}

We have presented the spectroscopic constants for 29 electronic states of $\mathrm{RuC}$ obtained using the FOCI method in Table I. Figures 1 through 4 show the potential curves of these electronic states, grouped according to their spin multiplicities. For every potential curve in the figures, single-point FOCI energy calculations were carried out from $1.3 \AA$ to $7.0 \AA$, with more points placed in the vicinity of the equilibrium geometry. Morse model function was used to fit the energy curves and then spectroscopic constants were obtained from the fitted model parameters. ${ }^{27}$ Although the dissociation energy $\left(D_{e}\right)$ of every state can also be obtained in this fashion, the values shown in Table I were actually obtained through a direct subtraction of the total energy of "supermolecular" $\mathrm{RuC}$ at the corresponding dissociation limits $(7 \AA)$ from the molecular energy at the equilibrium geometry. In general, this fitting approach was found to be able to provide accurate representations to all the discrete energy points, both in the vicinity of the equilibrium geometry and close to the dissociation limits.

As can be seen from Table I, the ${ }^{3} \Delta$ (1) state of RuC competes with the ${ }^{1} \Sigma^{+}$ state, which is believed to be the ground state of $\mathrm{RuC}$. In fact the two states are very close in energy as the level of theory increases. The ${ }^{3} \Delta$ (1) state has a bond length of $1.67 \AA$ at the FOCI level (smaller basis set), while the ${ }^{1} \Sigma^{+}$state has a slightly shorter both length (1.64 $\AA$ ). The energy difference between the ${ }^{3} \Delta(1)$ and ${ }^{1} \Sigma^{+}$states is about $0.47 \mathrm{eV}$ at the FOCI level but the ${ }^{1} \Sigma^{+}$state gets stabilized at a higher level. The ${ }^{1} \Delta$ 
state is found to lie at about $1435 \mathrm{~cm}^{-1}$ higher than the ${ }^{1} \Sigma^{+}$state at the FOCI level. We have provided all energy separations relative to the ${ }^{1} \Sigma^{+}$state in Table I since this is believed to be the ground state, and at a higher level this gets more stabilized.

The ${ }^{3} \Delta(1)$ and ${ }^{1} \Sigma^{+}$states must correlate into different dissociation limits, as confirmed in the figures. The ${ }^{3} \Delta(1)$ state dissociates into $\mathrm{Ru}$ and $\mathrm{C}$ atoms in their respective ground states, i.e.:

$$
\mathrm{RuC} \rightarrow \mathrm{Ru}\left({ }^{5} \mathrm{~F}\right)+\mathrm{C}\left({ }^{3} \mathrm{P}\right)
$$

This is also the case for all the other triplet, quintet and heptet states obtained in our calculations. On the other hand, the ${ }^{1} \Sigma^{+}$state and all the other singlet states correlate into the $\mathrm{Ru}\left[{ }^{3} \mathrm{~F}\left(4 d^{7} 5 s^{1}\right)\right]+\mathrm{C}\left({ }^{3} \mathrm{P}\right)$ limit. The energy difference between the two dissociation limits was calculated to be $0.90 \mathrm{eV}$ in the FOCI level, which is higher than the well-established experimental value of $0.78 \mathrm{eV}$ in Ref. 22. Consequently, it is evident that the ${ }^{1} \Sigma^{+}$state is not well represented by the FOCI method and should become stable by at least $0.12 \mathrm{eV}$. This is confirmed by higher-order computations that we have carried out. The dissociation energies of the ${ }^{3} \Delta(1)$ and ${ }^{1} \Sigma^{+}$states were found to be 6.23 and $6.66 \mathrm{eV}$, respectively indicating strong bonding between ruthenium and carbon atoms. In contrast to Shim et al. ${ }^{11}$, we find the ${ }^{7} \Sigma^{+}$state lies at $4.02 \mathrm{eV}$ above the ${ }^{3} \Delta(1)$ state, while Shim et $\mathrm{al}^{11}$ find this state at $1.58 \mathrm{eV}$ above the ${ }^{3} \Delta$ (1) state. Shim et al. have also found three other heptet states $\left({ }^{7} \Pi,{ }^{7} \Phi\right.$ and $\left.{ }^{7} \Delta\right)$ at $1.73,1.74$, and $1.97 \mathrm{eV}$, while the corresponding states are considerably higher in energy in our study. We believe that these discrepancies are most probably due to the limited HF/CI approach ${ }^{11}$ employed in the earlier study, while the current study uses 
the CASSCF/FOCI method. On the other hand, some low-lying quintet states were found to exist, in particular the ${ }^{5} \Pi(1)$ and ${ }^{5} \Delta$ (1) states lie at about 1.94 and $2.64 \mathrm{eV}$ above the ${ }^{3} \Delta$ (1) state, compared with the earlier work of Shim et al ${ }^{11}$ who have found that the ${ }^{5} \Pi$ (1) and ${ }^{5} \Delta$ (1) states lie at about 1.57 and $1.67 \mathrm{eV}$ above the ${ }^{3} \Delta$ (1) state. Overall, the low-spin states tend to be stabilized by electron correlation effects while the high spin states are stabilized by spin exchange energy. Thus at a lower level of theory, which includes electron correlation effects to a lesser degree, the higher spin states are favored. The lower spin states are favored at higher levels, as electron correlation effects are more accurately addressed at the higher levels.

As discussed before, our FOCI computations were followed by higher order computations of the low-lying electronic states at the FO+MRCI and MRSDCI levels. We invoked a harmonic fitting procedure for the spectroscopic constants at the higher level. The results at the (FO+MRCI) level are shown in table II together with the MRSDCI+Q energy separations. The primary difference between the FOCI results and the MRCI results is in the energy separations of the electronic states which are far more sensitive to dynamical electron correlation effects compared to the bond lengths and frequencies. A critical comparison of tables I and II reveals that the vibrational frequencies are quite similar, and the bond distances change by $0.01-0.05 \breve{A}$. However the major difference is in the energy separations. As can be seen from Table II, the energy separation of the ${ }^{1} \Sigma^{+}$state becomes less as the level of theory increases. At the highest level we still find the ${ }^{1} \Sigma^{+}$state to be $1323 \mathrm{~cm}^{-1}$ above the ${ }^{3} \Delta$ (1) state. Even a MRSDCI+Q with 18 million configurations could not yield the ${ }^{1} \Sigma^{+}$state to be the 
ground state. Hence inclusion of higher-order correlation effects, basis set improvements and spin-orbit effects could be attributed to the observed ${ }^{1} \Sigma^{+}$ground state. The energy separations of other excited states of RuC were lowered by $0.1 \sim 0.2$ $\mathrm{eV}$ at the MRSDCI+Q level, but no switchings of the relative order of electronic states were found. Thus we conclude that with the exception of the ${ }^{1} \Sigma^{+}$state overall trends of the electronic states in Table I should hold and the results in Table I should provide qualitative guidance to predict and assign new spectra of RuC.

Table III shows a comparison of our best-computed results with the experimental results of Langenberg et al. ${ }^{12}$ and Dabell et al. ${ }^{13}$ for the spectroscopic constants with the exception of dipole moments that come from the work of Steimle et al. ${ }^{15}$ As discussed before, the best possible fit of the observed R2PI spectra of Langenberg et al. ${ }^{12}$ was consistent with the ${ }^{1} \Sigma^{+}$ground state, with a bond length of 1.607 A, vibrational frequency of about $1100.0 \mathrm{~cm}^{-1}$ and $\omega_{\mathrm{e}} \chi_{\mathrm{e}}$ at about $5.3 \mathrm{~cm}^{-1}$. The first excited state of $\mathrm{RuC}$ was found to be ${ }^{3} \Delta$ state, with its three spin-orbit components lying at 76 to about $2044 \mathrm{~cm}^{-1}$ higher above the ground state. This state has a bond length of $1.635 \AA$, vibrational frequency of $1040 \mathrm{~cm}^{-1}$ and $\omega_{\mathrm{e}} \chi_{\mathrm{e}}$ of $4.7 \mathrm{~cm}^{-1}$. As seen from Table III, comparing our results with their observations, our fitted spectroscopic constants of the ${ }^{3} \Delta(1)$ and ${ }^{1} \Sigma^{+}$states agree very well with the above experimental results, the calculated bond lengths also indicate a similar trend but our values are systematically longer than the experimental values by $0.03 \AA$. The major difficulty that still remains is in the energy separations of low-lying states, which cannot be established as accurately as spectroscopic studies. Nevertheless this is not 
really surprising if we take into consideration the proximity of the two states involved. However, note that the energy separations of the excited states can alter up to 2000 $\mathrm{cm}^{-1}$ by spin-orbit coupling on $\mathrm{Ru}$.

In this year Steimle et al. ${ }^{15}$ reported the permanent electric dipole moments of the ${ }^{1} \Pi$ and ${ }^{1} \Sigma^{+}$states of RuC using high-resolution laser induced fluorescence spectroscopy. These results are included in Table III for comparison. Our calculated permanent dipole moment for the ${ }^{1} \Sigma^{+}$state is $4.21 \mathrm{D}$ at the FO+MRCI level compared to their measured value (4.09D). Thus higher-order methods generally yield reasonable dipole moments and thus the dipole moments of other excited triplet and quintet states should hold in Table III. However, an exception is that our calculated result for the first ${ }^{1} \Pi$ state at the FOCI level (4.5D) was much higher than the experimental result (3.31D). But the FO+MRCI method improved the dipole moment agreement only slightly in that our value is $4.39 \mathrm{D}$ at this level. This may be attributed partly to the longer bond length that is computed for the ${ }^{1} \Pi$ state. As indicated by Lengenberg et al., ${ }^{12}$ there may exist strong mixing between the several ${ }^{3} \Phi$ and ${ }^{1} \Phi$ states in this energy range due to spin-orbit coupling. Likewise there could be spin-orbit mixing of the $\Omega=1$ component of the ${ }^{1} \Pi$ state with other $\Omega=1$ states in the proximity. This could bring about the changes in the bond length and the dipole moments of the ${ }^{1} \Pi$ state.

The spin-orbit effects of RuC warrant discussion. The atomic spin-orbit splitting parameter of $\mathrm{Ru}$ is known to be about $1038 \mathrm{~cm}^{-1}$ as noted by Langenberg et al. ${ }^{12}$ The spin-orbit effects are expected to be quenched in states such as ${ }^{1} \Sigma^{+}$due to the 
strong involvement of the carbon orbitals in bonding which quenches the high-spin nature of $\mathrm{Ru}$ by paring up with carbon electrons. On the other hand, the splitting between the $\Omega$ components for higher $\Lambda$ states such as ${ }^{3} \Pi,{ }^{3} \Delta$, etc., should follow the $\mathrm{Ru}$ (4d) spin-orbit splitting. Indeed the spectroscopic study of Langenberg et al. ${ }^{12}$ confirms this. They find the ${ }^{3} \Pi_{2}-{ }^{3} \Pi_{0}$ splitting to be $579 \mathrm{~cm}^{-1}$, while the ${ }^{3} \Delta_{3}{ }^{3} \Delta_{1}$ spin-orbit splitting is $1820 \mathrm{~cm}^{-1}$. The mixing between other spin-orbit components is such that one can treat RuC as Hund's case (a) ${ }^{12}$ and thus there appears to be no compelling case to treat spin-orbit coupling in a variational manner for RuC. The only significant mixing would be that between ${ }^{3} \Pi_{1}$ and ${ }^{1} \Pi_{1}$ or ${ }^{3} \Delta_{2}$ and ${ }^{1} \Delta_{2}$ or ${ }^{3} \Phi_{3}$ and ${ }^{1} \Phi_{3}$ states, as these arise from the same electronic configurations and are energetically in the near proximity to each other. On the basis of this, we conclude that the spectroscopic constants listed in Table I can be treated within Hund's case (a) by identifying the states predominantly by their $\Lambda$ states and then obtaining the $\Omega$ components. The splitting among the $\Omega$ components for lower $\Lambda$ states is expected to be $\sim 600 \mathrm{~cm}^{-1}$, while the high $\Lambda$ states could undergo splitting up to $2000 \mathrm{~cm}^{-1}$. Thus the overall qualitative ordering of the electronic states must remain as in Table I.

Next we compare our results with those of Shim and coworkers ${ }^{11,14}$ who have carried out two studies on RuC. In their earlier all-electron study ${ }^{11}$ on RuC in 1987, Shim and her colleagues investigated 28 electronic states, which would have been the results suitable for a side-by-side comparison with our results. However, these earlier calculations were based on limited Hartree-Fock and valence CI methods, while most excited electronic states of RuC exhibit substantial multi-configurational features. 
This observation is confirmed by Table IV, in which the leading configurations of various electronic states of RuC obtained in our FOCI calculations are given. Even for those states with predominantly one leading configuration $(>80 \%)$, our results show some discrepancies with those of Shim et. al's ${ }^{11}$ earlier work. Some of these discrepancies have been discussed. Another sharp contrast is that while Shim and coworkers located 13 electronic states in the range of $1-2 \mathrm{eV}$ above the ground state, only four states are founding the same range in our current calculations in that energy gap. This is primarily due to the fact that the valence CI tends to crowd the electronic states as high spin states tend to be clustered together and thus the energy separations of these states are too close to the ground state at lower levels. On the other hand, at the HF/CI level of theory Shim and coworkers also found that the ${ }^{3} \Delta$ state was lower than the ${ }^{1} \Sigma^{+}$state.

Shim and Gingerich ${ }^{14}$ have carried out all-electron MRCI study on three lowest lying states of $\mathrm{RuC}$, the ${ }^{3} \Delta(1),{ }^{1} \Sigma^{+}$and ${ }^{1} \Delta$ (1) states, including perturbational relativistic corrections using a $(11 s 9 p 5 d 2 f / 4 s 3 p 1 d)$ basis set. They found the ground state to be the ${ }^{1} \Sigma^{+}$state with ${ }^{3} \Delta$ (1) lying at $912 \mathrm{~cm}^{-1}$ above it. In general their results agreed well with the experiments. When spin-orbit coupling was also taken into consideration, the energy difference between the ${ }^{3} \Delta_{3}$ and ${ }^{1} \Sigma^{+}$states lowered to $45 \mathrm{~cm}^{-1}$, in very good agreement with the experimental value of $76 \mathrm{~cm}^{-1}$. We have employed large basis sets and lower cutoff limit in our MRSDCI+Q calculations that included up to 18 million configurations, considerably larger than the computation of Shim and Gingerich. ${ }^{14}$ Yet the ${ }^{3} \Delta(1)$ and the ${ }^{1} \Sigma^{+}$states are quite close but the ${ }^{3} \Delta$ (1) state was 
slightly lower with a mere $0.164 \mathrm{eV}$ separation. This is a clear demonstration of the difficulties one may face in the study of transition metal complexes when even a qualitatively correct description of the system requires the use of large basis sets, high-level treatment of dynamic correlation and incorporation of relativistic effects. We conclude that the results reported by Shim and Gingerich $^{14}$ in 2000 are much improved compared to their previous study but the recent study includes only 3 states (without spin-orbit) and thus comparison with other higher excited states reported in our work is not feasible.

Table IV shows the leading configurations of the electronic states of RuC considered in this study. As can be seen from Table IV, the ground state of RuC is predominantly $1 \sigma^{2} 2 \sigma^{2} 1 \pi^{4} 1 \delta^{4}$ which suggests a predominantly closed-shell character for the $\mathrm{X}^{1} \Sigma^{+}$ground state. The $1 \delta$ orbital is purely $\mathrm{Ru}(4 \mathrm{~d})$ while the $1 \pi$ orbital is a mixture of $\mathrm{Ru}(4 \mathrm{~d} \pi)$ ad $\mathrm{C}(2 \mathrm{p} \pi)$. This is consistent with the back electron transfer from the $C(2 p)$ to $\mathrm{Ru}(4 \mathrm{~d} \pi)$ due to the dative bonding in $\mathrm{RuC}$. The $1 \sigma$ orbital is predominantly $\mathrm{C}(2 \mathrm{~s})$ while the $2 \sigma$ orbital is a mixture of $\mathrm{C}(2 \mathrm{p} \sigma)$ with $\mathrm{Ru}(4 \mathrm{~d} \sigma)$. The excited electronic states of $\mathrm{RuC}$ are considerably more complex, some with leading configurational weights as low as 28 to $60 \%$, as can be seen from Table IV. This clearly demonstrates the strong mixing of different states and high multi-reference characters of the excited electronic states of $\mathrm{RuC}$. Indeed it is this feature of $\mathrm{RuC}$, which makes it very challenging, as one has to include a large number of reference configurations with coefficient cutoff as small as 0.01 . This leads to combinatorial explosion of the number of configurations in the MRSDCI quite rapidly. 
Table V shows the Mulliken populations of several lowest-lying electronic states of RuC. Electronic populations of the $4 f$ orbital of ruthenium are generally negligible, and are thus not shown. As shown in Table V, for the ${ }^{3} \Delta$ (1) state, the $5 \mathrm{~s}$ population of $\mathrm{Ru}$ is about 0.830 , indicating an unpaired electron in the $5 s$ orbital of $\mathrm{Ru}$, with another unpaired electron in its $4 d$ orbital as inferred from the $4 \mathrm{~d}$ population. On the other hand, for the ${ }^{1} \Sigma^{+}$state, the $5 s$ orbital of $\mathrm{Ru}$ is almost zero. This can be interpreted as follows. The $\mathrm{Ru}(5 \mathrm{~s})$ orbital transfers all of its electron density to the carbon in its ${ }^{1} \Sigma^{+}$state to create $\mathrm{Ru}^{+}-\mathrm{C}^{-}$ionic bonding. This is followed by back transfer of $\mathrm{C}(2 \mathrm{p} \pi)$ electronic density to the $\mathrm{Ru}(4 \mathrm{~d} \pi)$. The back transfer from $\mathrm{C}$ to $\mathrm{Ru}$ is about 0.69 electrons so as to result in an excess $4 d$ population of 0.69 in the $R u(4 d)$. Consequently, we have dative bonding between $\mathrm{Ru}$ and $\mathrm{C}$, where ionic part arises from electron transfer from $\mathrm{Ru}(5 \mathrm{~s})$ to $\mathrm{C}(2 \mathrm{p})$ and the covalent part comes from back transfer from $\mathrm{C}(2 \mathrm{p})$ to $\mathrm{Ru}(5 \mathrm{p})$ through a $\mathrm{Ru}(4 \mathrm{~d} \pi)-\mathrm{C}(2 \mathrm{p} \pi)$ back bonding. An interesting feature is that the $5 \mathrm{p}$ populations on $\mathrm{Ru}$ for most of the electronic states are 0.1 to 0.3 . This suggests considerable involvement of the $5 \mathrm{p}$ orbital in those electronic states. The $2 s$ and $2 p$ orbitals of carbon seem to play a similar role in the two states, with the $2 p$ population slightly higher in the ${ }^{1} \Sigma^{+}$state. A striking feature in Table $\mathrm{V}$ is the large variation in the $\mathrm{Ru}(4 \mathrm{~d})$ population. Indeed this is the primary contrast between the ${ }^{3} \Delta$ and ${ }^{1} \Sigma^{+}$states. As seen from Table $\mathrm{V}$, the ${ }^{1} \Sigma^{+}$state of RuC has a $4 \mathrm{~d}$ population of 7.69 , which is substantially larger than the corresponding value for the ${ }^{3} \Delta$ state. A similar trend is noted for the ${ }^{1} \Delta$ state. The ${ }^{3} \Sigma^{-}$and ${ }^{5} \Pi$ states exhibit $4 \mathrm{~d}$ populations that are almost over 1.1-1.5 electron deficient in the $4 \mathrm{~d}$ suggesting that 
these states arise from a different electronic configuration of $\mathrm{Ru}$, viz., $4 \mathrm{~d}^{6} 5 \mathrm{~s}^{2}$ compared to other electronic states which arise from $4 d^{7} 5 s^{1}$.

The total $\mathrm{Ru}$ populations in most of the electronic states are less than their neutral atomic populations suggesting that there is considerable charge transfer from $\mathrm{Ru}$ to $\mathrm{C}$. Hence the bonding of ruthenium atom to carbon atom is considerably ionic, and the covalent part is facilitated by back transfer from $\mathrm{C}$ to $\mathrm{Ru}$ by the overlap of the $\mathrm{C} 2 p$ orbitals with the $\mathrm{Ru} 4 d$ orbitals leading to two $p-d \pi$ and one $p-d \sigma$ bonds. In Fig. 5 and Fig. 6 we have shown the electronic difference densities of RuC in the ${ }^{3} \Delta$ (1) and ${ }^{1} \Sigma^{+}$states relative to ruthenium and carbon atoms respectively, which also confirm our observations. The bonding is dative in nature as confirmed by the electron density contours, where $\mathrm{Ru}$ donates electron density to $\mathrm{C}$ forming $\mathrm{Ru}^{+} \mathrm{C}^{-}$bond followed by back-donation of electron density from $\mathrm{C}$ to the $\mathrm{Ru}(d \pi)$ orbital. This is a dative mechanism of electron transfer followed by back transfer. Note that the extent of charge transfer is stronger in the ${ }^{1} \Sigma^{+}$sate compared to the ${ }^{3} \Delta$ state as seen from comparing figures 5 and 6 .

\section{CONCLUSIONS}

Spectroscopic constants and potential energy curves of 29 low-lying electronic states of ruthenium carbide have been investigated at the FOCI level. A few lower electronic states were further studied at the FO+MRCI and MRSDCI levels that included up to 18 million configurations. Our computed spectroscopic constants of the

low-lying electronic states of $\mathrm{RuC}$ were in good agreement with the results of 
Langenberg et al. ${ }^{12}$ and DaBell et al. ${ }^{13}$ As the level of theory increases we found the ${ }^{1} \Sigma^{+}$state to be more stabilized relative to the ${ }^{3} \Delta$ (1) state, which becomes lower at the FOCI level. Our computations supported the previous assignments ${ }^{11,12}$ of the observed bands, and we have also predicted the spectroscopic constants of a number of new electronic states that are yet to be observed. Our computed permanent dipole moment of the ground state of $\mathrm{RuC}$ is in excellent agreement with the experimental value reported by Steimle et al, ${ }^{15}$ although the dipole moment of the excited ${ }^{1} \Pi$ state that we compute differs from the experiment primarily due to our computed longer bond length for this excited state. The nature of bonding in the molecule was also analyzed in light of Mulliken population and electron difference density of the lower-lying states. It was found that the bonding is predominantly ionic with $\mathrm{Ru}^{+} \mathrm{C}^{-}$ polarity with considerable electron back transfer from $\mathrm{C}$ to $\mathrm{Ru}$ through the $\operatorname{Ru}(4 \mathrm{~d} \pi)-\mathrm{C}(2 \mathrm{p} \pi)$ bonding. That is, the $\operatorname{Ru}(5 \mathrm{~s})$ transfers all of its electron density to the carbon in its ${ }^{1} \Sigma^{+}$state to create $\mathrm{Ru}^{+}-\mathrm{C}^{-}$ionic bonding This is followed by a back transfer of $C(2 p)$ electronic density to the $R u(4 d \pi)$ orbital. The back transfer from $\mathrm{C}(2 \mathrm{p})$ to $\mathrm{Ru}(4 \mathrm{~d})$ was inferred as 0.69 electrons on the basis of the Mulliken populations. Consequently, we found evidence of dative bonding between $\mathrm{Ru}$ and $\mathrm{C}$, where ionic part arises from electron transfer from $\mathrm{Ru}(5 \mathrm{~s})$ to $\mathrm{C}(2 \mathrm{p})$ and the covalent part comes from back transfer from $C(2 p)$ to $\mathrm{Ru}(5 p)$ through a $\mathrm{Ru}(4 \mathrm{~d} \pi)-\mathrm{C}(2 \mathrm{p} \pi)$ back bonding. 


\section{ACKNOWLEDGMENT}

This research was supported by the U.S. Department of Energy under Grant No. DEFG2-86ER13558. The work at LLNL was performed under the auspices of US Department of Energy by the University of California under Contract No.W-7405-Eng-48.

\section{REFERENCES}

${ }^{1}$ H. Tan, M. Liao, and K. Balasubramanian, Chem. Phys. Lett. 280, 423 (1997).

${ }^{2}$ D. Majumdar and K. Balasubramanian, Chem. Phys. Lett. 284, 273 (1998).

${ }^{3}$ D. Tzeli and A. Mavridis, J. Chem. Phys. 116, 4901 (2002).

${ }^{4}$ K. Balasubramanian, J. Chem. Phys. 112, 7425 (2000).

${ }^{5}$ D. Majumdar and K. Balasubramanian, Mol. Phys. 101, 1369 (2003).

${ }^{6}$ I. Shim and K. A. Gingerich, J. Chem. Phys. 106, 8093 (1997).

${ }^{7}$ R. Scullman and B. Thelin, Phys. Scr. 3, 19 (1971).

${ }^{8}$ R. Scullman and B. Thelin, Phys. Scr. 5, 201 (1972).

${ }^{9}$ N. S. McIntyre, A. V. Auwera-Mahieu, and J. Drowart, Trans. Faraday Soc. 64, 3006 (1968).

${ }^{10}$ K. A. Gingerich, Chem. Phys. Lett. 25, 523 (1974).

${ }^{11}$ I. Shim, H. C. Finkbeiner and K. A. Gingerich, J. Phys. Chem. 91, 3171 (1987).

12 J. D. Langenberg, R. S. DaBell, L. Shao, D. Dreessen, and M. D. Morse, J. Chem. Phys. 109, 7863 (1998).

${ }^{13}$ R. S. DaBell, R. G. Meyer, and M. D. Morse, J. Chem. Phys. 114, 2938 (2001).

${ }^{14}$ I. Shim and K. A. Gingerich, Chem. Phys. Lett. 317, 338 (2000).

${ }^{15}$ T. C. Steimle, W. L. Virgo, and J. M. Brown, J. Chem. Phys. 118, 2620 (2002).

${ }^{16}$ A. V. Auwera-Mahieu, R. Peeters, N. S. McIntyre, and J. Drowart, Trans. Faraday Soc. 66, 809 (1970). 
${ }^{17}$ S. R. Langhoff and E. R. Davidson, Int. J. Quantum Chem. S9, 183 (1975).

${ }^{18}$ L. A. LaJohn, P. A. Christiansen, R. B. Ross, T. Atashroo, and W. C. Ermler, J. Chem. Phys. 87, 2812 (1987).

${ }^{19}$ R. Guo and K. Balasubramanian, J. Chem. Phys. 118, 142 (2003).

${ }^{20}$ L. F. Pacios and P. A. Christiansen, J. Chem. Phys. 82, 2664 (1985).

${ }^{21}$ T. H. Dunning, Jr. and P. J. Hay, in Methods of Electronic Structure Theory, Vol. 1 (Plenum Press, New York, 1977).

${ }^{22}$ C. E. Moore, Table of Atomic Energy Levels (National Bureau of Standards, 35/V Circular 467, Washington, D.C., 1971).

${ }^{23}$ The major authors of ALCHEMY II are B. Liu, B. Lengsfield, and M. Yoshimine.

${ }^{24}$ K. Balasubramanian, Chem. Phys. Lett. 127, 585 (1986).

${ }^{25}$ K. Balasubramanian, Modified Enhanced Version of ALCHEMY II, 2002.

${ }^{26}$ M. W. Schmidt, K. K. Baldridge, J. A. Boatz, S. T. Elbert, M. S. Gordon, J. J. Jensen, S. Koseki, N. Matsunaga, K. A. Nguyen, S. Su, T. L. Windus, M. Dupuis, and J. A. Montgomery, J. Comput. Chem. 14, 1347 (1993).

${ }^{27}$ G. Herzberg, Molecular Spectra and Molecular Structure: Spectra of Diatomic Molecules, (van Nostrand Reinhold, New York, 1950). 

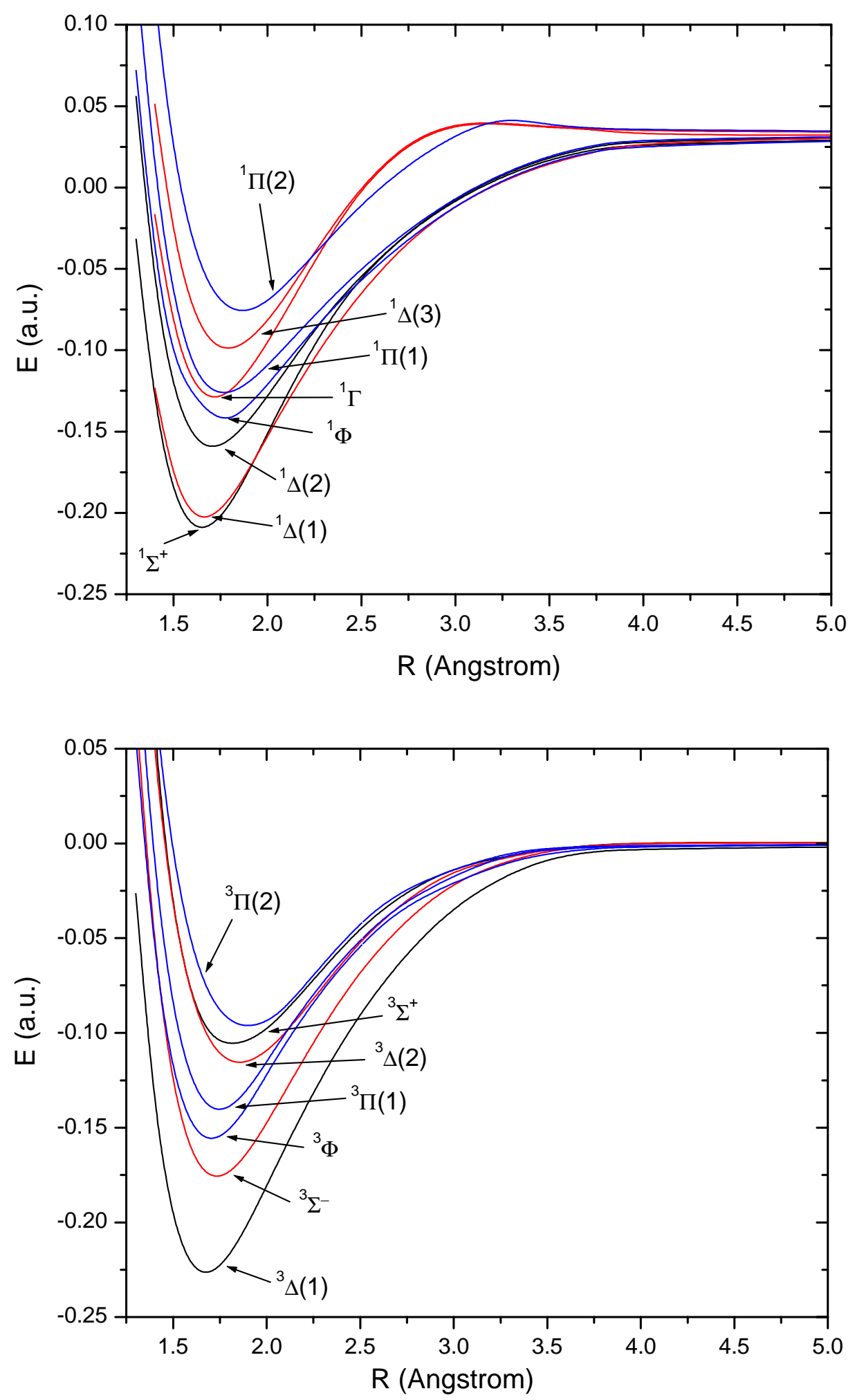

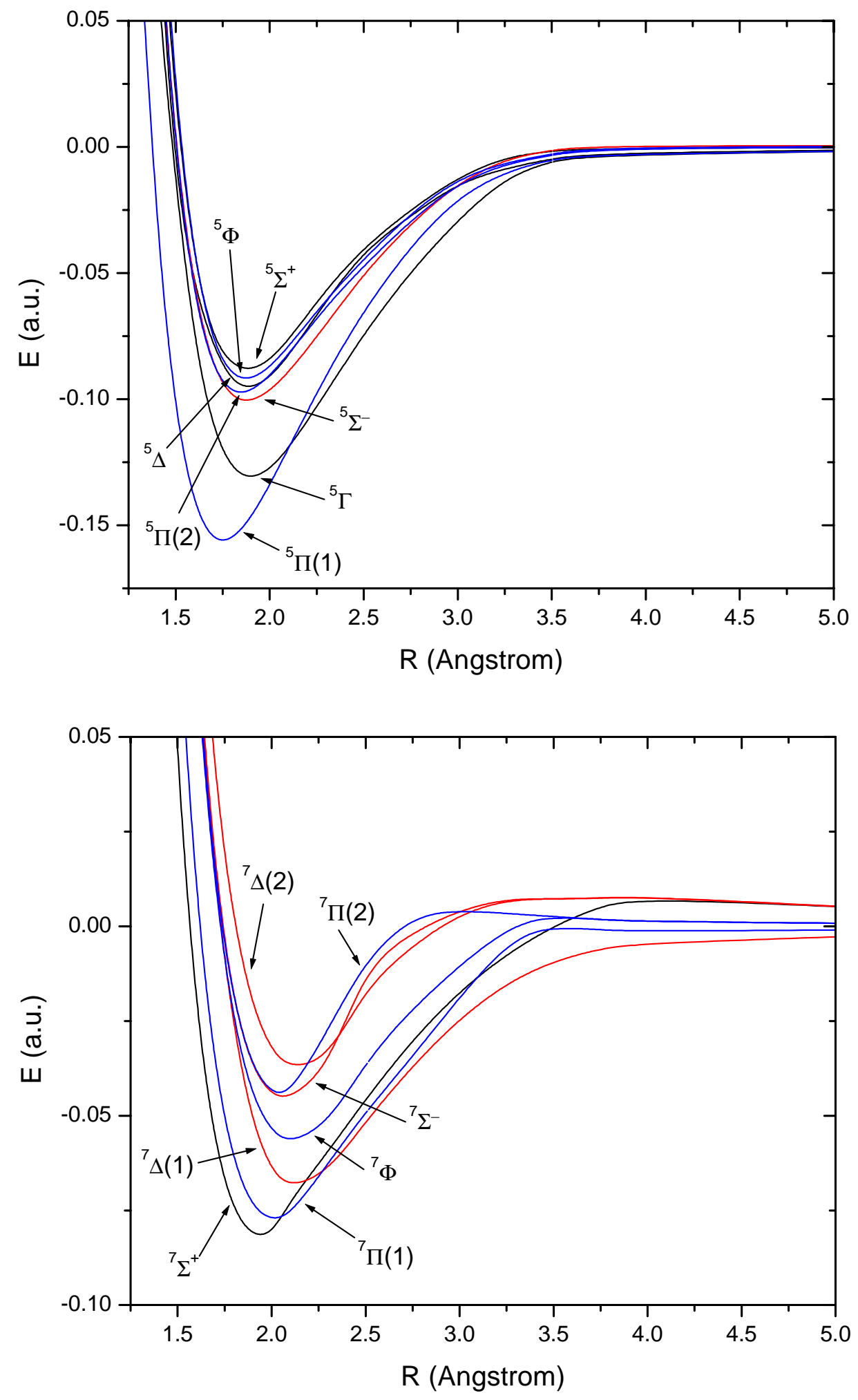

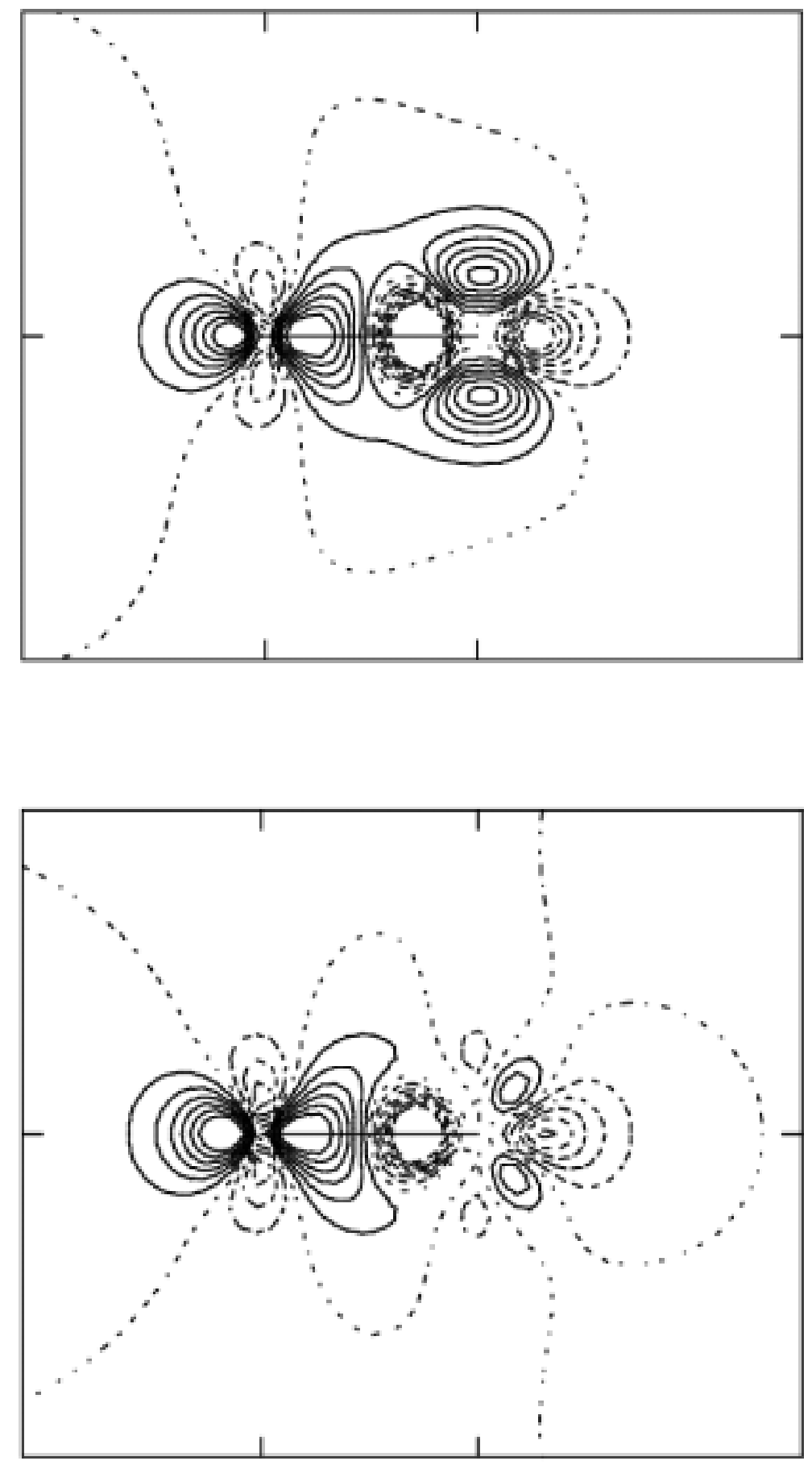
TABLE I. Fitted spectroscopic constants of the potential curves of various electronic states of ruthenium carbide calculated at FOCI level

\begin{tabular}{|c|c|c|c|c|c|c|}
\hline $\begin{array}{c}\text { Assignm } \\
\text { ent }\end{array}$ & $\mathrm{T}_{\mathrm{e}}\left(\mathrm{cm}^{-1}\right)$ & $r_{e}(\AA)$ & $\begin{array}{c}\omega_{\mathrm{e}} \\
\left(\mathrm{cm}^{-1}\right)\end{array}$ & $\begin{array}{c}\omega_{\mathrm{e}} \chi_{\mathrm{e}} \\
\left(\mathrm{cm}^{-1}\right)\end{array}$ & $\mathrm{D}_{\mathrm{e}}(\mathrm{eV})$ & $\mu_{\mathrm{e}}(\mathrm{D})$ \\
\hline${ }^{1} \Sigma^{+}$ & 0 & 1.642 & 1082 & 4.6 & 6.664 & 4.551 \\
\hline${ }^{3} \Delta(1)$ & -3767 & 1.666 & 1023 & 4.9 & 6.227 & 1.492 \\
\hline${ }^{1} \Delta(1)$ & 1435 & 1.653 & 1048 & 5.2 & 6.486 & 0.657 \\
\hline${ }^{3} \Sigma^{-}$ & 7468 & 1.724 & 930 & 4.9 & 4.834 & 0.594 \\
\hline${ }^{1} \Delta(2)$ & 11090 & 1.697 & 924 & 5.7 & 5.289 & 3.822 \\
\hline${ }^{3} \Phi$ & 11832 & 1.696 & 945 & 4.7 & 4.293 & 4.538 \\
\hline${ }^{5} \Pi(1)$ & 11864 & 1.741 & 873 & 5.2 & 4.288 & 2.439 \\
\hline${ }^{1} \Phi$ & 15082 & 1.766 & 828 & 1.4 & 4.793 & 4.553 \\
\hline${ }^{3} \Pi(1)$ & 15243 & 1.738 & 914 & 4.7 & 3.870 & 4.850 \\
\hline${ }^{5} \Gamma$ & 17526 & 1.890 & 732 & 4.3 & 3.587 & 2.209 \\
\hline${ }^{1} \Gamma$ & 17728 & 1.710 & 970 & 4.6 & 4.466 & 0.284 \\
\hline${ }^{1} \Pi(1)$ & 18445 & 1.758 & 814 & 5.5 & 4.377 & 4.413 \\
\hline${ }^{3} \Delta(2)$ & 20833 & 1.848 & 719 & 3.4 & 3.177 & 1.853 \\
\hline${ }^{3} \Sigma^{+}$ & 22954 & 1.806 & 711 & 6.4 & 2.914 & 3.052 \\
\hline${ }^{5} \Sigma^{-}$ & 24140 & 1.868 & 699 & 5.5 & 2.767 & 0.576 \\
\hline${ }^{1} \Delta(3)$ & 24398 & 1.782 & 865 & 4.2 & 3.638 & 1.864 \\
\hline${ }^{5} \Pi(2)$ & 24833 & 1.837 & 744 & 6.9 & 2.680 & 2.208 \\
\hline${ }^{3} \Pi(2)$ & 25043 & 1.893 & 674 & 2.3 & 2.654 & 4.366 \\
\hline${ }^{5} \Delta$ & 25527 & 1.886 & 679 & 2.6 & 2.595 & 2.308 \\
\hline${ }^{5} \Phi$ & 26067 & 1.864 & 730 & 6.5 & 2.527 & 2.379 \\
\hline${ }^{5} \Sigma^{+}$ & 26842 & 1.867 & 708 & 5.2 & 2.431 & 2.377 \\
\hline${ }^{7} \Sigma^{+}$ & 28616 & 1.918 & 624 & 6.0 & 2.211 & 2.905 \\
\hline${ }^{7} \Pi(1)$ & 29423 & 2.000 & 594 & 4.6 & 2.111 & 3.616 \\
\hline${ }^{1} \Pi(1)$ & 29600 & 1.857 & 757 & 4.0 & 2.994 & 3.969 \\
\hline
\end{tabular}




\begin{tabular}{ccccccc}
${ }^{7} \Delta(1)$ & 31012 & 2.132 & 551 & 4.0 & 1.915 & 2.682 \\
${ }^{7} \Phi$ & 33907 & 2.098 & 547 & 3.8 & 1.556 & 3.829 \\
${ }^{7} \Sigma^{-}$ & 36278 & 2.054 & 624 & 3.2 & 1.261 & 0.784 \\
${ }^{7} \Pi(2)$ & 36730 & 2.029 & 656 & 3.4 & 1.106 & 2.324 \\
${ }^{7} \Delta(2)$ & 37658 & 2.207 & 424 & 3.3 & 1.090 & 2.046 \\
\hline
\end{tabular}


TABLE II. Spectroscopic constants of the lower-lying electronic states of $\mathrm{RuC}$ at the FO+MRCI level and at the MRSDCI+Q level with the larger basis sets $(5 s 4 p 4 d 2 f+$

\begin{tabular}{|c|c|c|c|c|c|c|c|}
\hline \multirow[b]{2}{*}{ State } & \multirow[b]{2}{*}{$\begin{array}{l}\mathrm{r}_{\mathrm{e}} \\
(\AA)\end{array}$} & \multicolumn{3}{|c|}{$\mathrm{FO}+\mathrm{MRCI}$} & \multicolumn{2}{|c|}{$\mathrm{MRSDCI}+\mathrm{Q}$} & \multirow{2}{*}{$\begin{array}{c}\text { Exp. } \\
\mathrm{T}_{\mathrm{e}} \\
\left(\mathrm{cm}^{-1}\right)\end{array}$} \\
\hline & & $\begin{array}{c}\omega_{\mathrm{e}} \\
\left(\mathrm{cm}^{-1}\right)\end{array}$ & $\begin{array}{c}\mathrm{T}_{\mathrm{e}} \\
\left(\mathrm{cm}^{-1}\right)\end{array}$ & $\begin{array}{l}\text { Dipole } \\
\text { (D) }\end{array}$ & $\begin{array}{c}\mathrm{T}_{\mathrm{e}} \\
\left(\mathrm{cm}^{-1}\right)\end{array}$ & $\begin{array}{l}\text { Dipole } \\
\text { (D) }\end{array}$ & \\
\hline${ }^{1} \Sigma^{+}$ & 1.634 & 1085 & 0 & 4.208 & 0 & 4.221 & 0 \\
\hline${ }^{3} \Delta(1)$ & 1.667 & 1034 & -2766 & 1.522 & -1323 & 1.678 & 726 \\
\hline${ }^{1} \Delta(1)$ & 1.649 & 1052 & 2331 & 0.712 & 3581 & 0.905 & 5678 \\
\hline${ }^{3} \Phi$ & 1.692 & 949 & 12220 & 4.314 & 12049 & 4.463 & 13429 \\
\hline${ }^{5} \Pi(1)$ & 1.740 & 849 & 12647 & 2.454 & 13469 & 2.683 & - \\
\hline${ }^{1} \Phi$ & 1.714 & 818 & 14954 & 4.177 & 14494 & 4.311 & 16196 \\
\hline${ }^{5} \Gamma$ & 1.894 & 694 & 18229 & 2.313 & 19588 & 2.602 & - \\
\hline
\end{tabular}


TABLE III. Comparison of theoretical results and experimental values ${ }^{\mathrm{a}}$.

\begin{tabular}{|c|c|c|c|c|c|c|c|c|}
\hline \multirow{2}{*}{ State $^{b}$} & \multicolumn{2}{|c|}{$r_{e}(\AA)$} & \multicolumn{2}{|c|}{$\omega_{\mathrm{e}}\left(\mathrm{cm}^{-1}\right)$} & \multicolumn{2}{|c|}{$\mathrm{T}_{\mathrm{e}}\left(\mathrm{cm}^{-1}\right)$} & \multicolumn{2}{|c|}{ Dipole (D) } \\
\hline & Theo. & Exp. & Theo. & Exp. & Theo. & Exp. & Theo. & Exp. $^{\mathrm{c}}$ \\
\hline $\mathrm{X}^{1} \Sigma^{+}$ & 1.634 & 1.608 & 1085 & 1100 & 0 & 0 & 4.208 & 4.09 \\
\hline${ }^{3} \Delta(1)$ & 1.667 & 1.635 & 1034 & 1038 & -1323 & 728 & 1.522 & - \\
\hline${ }^{1} \Delta(1)$ & 1.649 & 1.621 & 1052 & 1068 & 3581 & 5679 & 0.712 & - \\
\hline${ }^{3} \Phi$ & 1.692 & 1.662 & 949 & 951 & 12049 & 13999 & 4.314 & - \\
\hline${ }^{5} \Pi(1)$ & 1.740 & - & 849 & - & 13469 & - & 2.454 & - \\
\hline${ }^{1} \Phi$ & 1.714 & 1.667 & 818 & 919 & 14954 & 16195 & 4.177 & - \\
\hline${ }^{3} \Pi(1)$ & 1.738 & 1.658 & 914 & 972 & 15243 & 13428 & 4.850 & - \\
\hline${ }^{1} \Pi(1)$ & 1.758 & 1.679 & 814 & 889 & 18445 & 18086 & 4.39 & 3.31 \\
\hline${ }^{5} \Gamma$ & 1.894 & - & 694 & - & 18229 & - & 2.313 & - \\
\hline
\end{tabular}

${ }^{\text {a }}$ Experimental values are $J$-averaged values from DaBell et al.

${ }^{\mathrm{b}}$ Theoretical results at the best level of theory.

${ }^{\mathrm{c}}$ Experimental values of Steimle et al. 
TABLE IV. Leading configurations of various electronic states of RuC at the FOCI level

\begin{tabular}{|c|c|}
\hline Assignment & Leading configurations $^{\mathrm{a}}$ \\
\hline${ }^{1} \Sigma^{+}$ & $1 \sigma^{2} 2 \sigma^{2} 1 \pi^{4} 1 \delta^{4}(82)$ \\
\hline${ }^{3} \Delta(1)$ & $1 \sigma^{2} 2 \sigma^{2} 3 \sigma^{1} 1 \pi^{4} 1 \delta^{3}(81)$ \\
\hline${ }^{1} \Delta(1)$ & $1 \sigma^{2} 2 \sigma^{2} 3 \sigma^{1} 1 \pi^{4} 1 \delta^{3}(81)$ \\
\hline${ }^{3} \Sigma^{-}$ & $1 \sigma^{2} 2 \sigma^{2} 3 \sigma^{2} 1 \pi^{4} 1 \delta^{2}(71)$ \\
\hline${ }^{1} \Delta(2)$ & $1 \sigma^{2} 2 \sigma^{2} 4 \sigma^{1} 1 \pi^{4} 1 \delta^{3}(37), 1 \sigma^{2} 2 \sigma^{2} 3 \sigma^{1} 1 \pi^{4} 1 \delta^{3}(17), 1 \sigma^{2} 2 \sigma^{2} \sigma_{\mathrm{v}} 11 \pi^{4} 1 \delta^{3}(6)$ \\
\hline${ }^{3} \Phi$ & $1 \sigma^{2} 2 \sigma^{2} 1 \pi^{4} 2 \pi^{1} 1 \delta^{3}(80)$ \\
\hline${ }^{5} \Pi(1)$ & $1 \sigma^{2} 2 \sigma^{2} 3 \sigma^{1} 1 \pi^{4} 2 \pi^{1} 1 \delta^{2}(81)$ \\
\hline${ }^{1} \Phi$ & $1 \sigma^{2} 2 \sigma^{2} 1 \pi^{4} 2 \pi^{1} 1 \delta^{3}(63)$ \\
\hline${ }^{3} \Pi(1)$ & $1 \sigma^{2} 2 \sigma^{2} 1 \pi^{4} 2 \pi^{1} 1 \delta^{3}(69)$ \\
\hline${ }^{5} \Gamma$ & $1 \sigma^{2} 2 \sigma^{2} 3 \sigma^{1} 1 \pi^{3} 2 \pi^{1} 1 \delta^{3}(73)$ \\
\hline${ }^{1} \Gamma$ & $1 \sigma^{2} 2 \sigma^{2} 3 \sigma^{2} 1 \pi^{4} 1 \delta^{2}(69), 1 \sigma^{2} 2 \sigma^{2} 3 \sigma^{1} 4 \sigma^{1} 1 \pi^{4} 1 \delta^{2}(5)$ \\
\hline${ }^{1} \Pi(1)$ & $1 \sigma^{2} 2 \sigma^{2} 1 \pi^{4} 2 \pi^{1} 1 \delta^{3}(55)$ \\
\hline \multirow[t]{2}{*}{${ }^{3} \Delta(2)$} & $1 \sigma^{2} 2 \sigma^{2} 3 \sigma^{1} 1 \pi^{3} 2 \pi^{1} 1 \delta^{3}(28), 1 \sigma^{2} 2 \sigma^{1} 3 \sigma^{2} 1 \pi^{4} 1 \delta^{3}(24)$ \\
\hline & $1 \sigma^{2} 2 \sigma^{1} 3 \sigma^{1} 4 \sigma^{1} 1 \pi^{4} 1 \delta^{3}(7), 1 \sigma^{2} 2 \sigma^{2} 4 \sigma^{1} 1 \pi^{4} 1 \delta^{3}(6)$ \\
\hline${ }^{3} \Sigma^{+}$ & $1 \sigma^{2} 2 \sigma^{1} 3 \sigma^{1} 1 \pi^{4} 1 \delta^{4}(36), 1 \sigma^{2} 2 \sigma^{2} 1 \pi^{3} 2 \pi^{1} 1 \delta^{4}(26)$ \\
\hline \multirow[t]{2}{*}{${ }^{5} \Sigma^{-}$} & $1 \sigma^{2} 2 \sigma^{2} 3 \sigma^{1} 4 \sigma^{1} 1 \pi^{4} 1 \delta^{2}(45), 1 \sigma^{2} 2 \sigma^{2} 3 \sigma^{2} 1 \pi^{3} 2 \pi^{1} 1 \delta^{2}(13)$ \\
\hline & $1 \sigma^{2} 2 \sigma^{2} 3 \sigma^{1} 1 \pi^{3} 2 \pi^{1} 1 \delta^{3}(8)$ \\
\hline${ }^{1} \Delta(3)$ & $1 \sigma^{2} 2 \sigma^{2} 4 \sigma^{1} 1 \pi^{4} 1 \delta^{3}(47), 1 \sigma^{2} 2 \sigma^{2}\left(8 a_{1}\right)^{1} 1 \pi^{4} 1 \delta^{3}(5)$ \\
\hline${ }^{5} \Pi(2)$ & $1 \sigma^{2} 2 \sigma^{1} 3 \sigma^{1} 1 \pi^{4} 2 \pi^{1} 1 \delta^{3}(43), 1 \sigma^{2} 2 \sigma^{1} 3 \sigma^{2} 1 \pi^{4} 2 \pi^{1} 1 \delta^{2}(25)$ \\
\hline \multirow[t]{2}{*}{${ }^{3} \Pi(2)$} & $1 \sigma^{2} 2 \sigma^{2} 4 \sigma^{1} 1 \pi^{4} 2 \pi^{1} 1 \delta^{2}(30), 1 \sigma^{2} 2 \sigma^{2} 4 \sigma^{1} 1 \pi^{3} 1 \delta^{4}(10)$ \\
\hline & $1 \sigma^{2} 2 \sigma^{2} 3 \sigma^{1} 1 \pi^{4} 2 \pi^{1} 1 \delta^{2}(9)$ \\
\hline${ }^{5} \Delta(2)$ & $1 \sigma^{2} 2 \sigma^{2} 3 \sigma^{1} 1 \pi^{3} 2 \pi^{1} 1 \delta^{3}(82)$ \\
\hline${ }^{5} \Phi$ & $1 \sigma^{2} 2 \sigma^{1} 3 \sigma^{1} 1 \pi^{4} 2 \pi^{1} 1 \delta^{3}(59)$ \\
\hline${ }^{5} \Sigma^{+}$ & $1 \sigma^{2} 2 \sigma^{2} 3 \sigma^{1} 1 \pi^{3} 2 \pi^{1} 1 \delta^{3}(68), 1 \sigma^{2} 2 \sigma^{2} 1 \pi^{4} 2 \pi^{2} 1 \delta^{2}(5)$ \\
\hline${ }^{7} \Sigma^{+}$ & $1 \sigma^{2} 2 \sigma^{1} 3 \sigma^{1} 1 \pi^{4} 2 \pi^{2} 1 \delta^{2}(65), 1 \sigma^{2} 2 \sigma^{1} 3 \sigma^{1} 1 \pi^{3} 2 \pi^{3} 1 \delta^{2}(12)$ \\
\hline${ }^{7} \Pi(1)$ & $1 \sigma^{2} 2 \sigma^{2} 3 \sigma^{1} 1 \pi^{3} 2 \pi^{2} 1 \delta^{2}(77)$ \\
\hline
\end{tabular}


${ }^{1} \Pi(2) \quad 1 \sigma^{2} 2 \sigma^{2} 4 \sigma^{1} 1 \pi^{4} 2 \pi^{1} 1 \delta^{2}(28), 1 \sigma^{2} 2 \sigma^{2} 4 \sigma^{1} 1 \pi^{3} 1 \delta^{4}(10)$,

$$
1 \sigma^{2} 2 \sigma^{2} 3 \sigma^{1} 1 \pi^{4} 2 \pi^{1} 1 \delta^{2}(6)
$$

${ }^{7} \Delta(1) \quad 1 \sigma^{2} 2 \sigma^{2} 3 \sigma^{1} 1 \pi^{2} 2 \pi^{2} 1 \delta^{3}(61), 1 \sigma^{2} 2 \sigma^{1} 3 \sigma^{1} 4 \sigma^{1} 1 \pi^{3} 2 \pi^{1} 1 \delta^{3}(20)$

${ }^{7} \Phi \quad 1 \sigma^{2} 2 \sigma^{1} 3 \sigma^{1} 1 \pi^{3} 2 \pi^{2} 1 \delta^{3}(75)$

${ }^{7} \Sigma^{-} \quad 1 \sigma^{2} 2 \sigma^{2} 3 \sigma^{1} 4 \sigma^{1} 1 \pi^{3} 2 \pi^{1} 1 \delta^{2}(57), 1 \sigma^{2} 2 \sigma^{1} 3 \sigma^{2} 4 \sigma^{1} 1 \pi^{3} 2 \pi^{1} 1 \delta^{2}(9)$,

$1 \sigma^{2} 2 \sigma^{2} 3 \sigma^{2} 1 \pi^{2} 2 \pi^{2} 1 \delta^{2}(8)$

${ }^{7} \Pi(2) \quad 1 \sigma^{2} 2 \sigma^{1} 3 \sigma^{1} 1 \pi^{3} 2 \pi^{2} 1 \delta^{3}(49), 1 \sigma^{2} 2 \sigma^{1} 3 \sigma^{1} 4 \sigma^{1} 1 \pi^{4} 2 \pi^{1} 1 \delta^{2}(25)$

${ }^{7} \Delta(2) \quad 1 \sigma^{2} 2 \sigma^{1} 3 \sigma^{1} 4 \sigma^{1} 1 \pi^{3} 2 \pi^{1} 1 \delta^{3}(40), 1 \sigma^{2} 2 \sigma^{1} 3 \sigma^{2} 1 \pi^{2} 2 \pi^{2} 1 \delta^{3}(14)$,

$1 \sigma^{2} 2 \sigma^{2} 3 \sigma^{1} 1 \pi^{2} 2 \pi^{2} 1 \delta^{3}(9), 1 \sigma^{2} 2 \sigma^{1} 3 \sigma^{1} 4 \sigma^{1} 1 \pi^{2} 2 \pi^{2} 1 \delta^{3}(7)$,

$1 \sigma^{2} 2 \sigma^{2} 4 \sigma^{1} 1 \pi^{2} 2 \pi^{2} 1 \delta^{3}(5)$

\footnotetext{
${ }^{\mathrm{a}}$ Numbers in parenthesis are the percentage of the configurations.
} 
TABLE V. Mulliken populations of the lowest electronic states of RuC.

\begin{tabular}{cccccccc}
\hline \multirow{2}{*}{ State } & \multicolumn{3}{c}{$\mathrm{Ru}$} & & \multicolumn{3}{c}{$\mathrm{C}$} \\
\cline { 2 - 4 } \cline { 6 - 8 } & $s$ & $p$ & $d$ & & $s$ & $p$ & $d$ \\
\hline${ }^{1} \Sigma^{+}$ & 2.005 & 6.057 & 7.690 & & 1.830 & 2.303 & 0.047 \\
${ }^{3} \Delta(1)$ & 2.830 & 6.142 & 6.899 & & 1.800 & 2.202 & 0.073 \\
${ }^{1} \Delta(1)$ & 2.873 & 6.162 & 6.858 & & 1.787 & 2.195 & 0.072 \\
${ }^{3} \Sigma^{-}$ & 3.615 & 6.135 & 6.203 & & 1.780 & 2.144 & 0.061 \\
${ }^{1} \Delta(2)$ & 2.729 & 6.065 & 7.041 & & 1.794 & 2.248 & 0.056 \\
${ }^{3} \Phi$ & 2.053 & 6.327 & 7.295 & & 1.771 & 2.413 & 0.061 \\
${ }^{5} \Pi(1)$ & 2.840 & 6.355 & 6.571 & & 1.790 & 2.315 & 0.060 \\
${ }^{1} \Phi$ & 2.027 & 6.230 & 7.423 & & 1.761 & 2.432 & 0.056 \\
\hline \hline
\end{tabular}




\section{Figure Captions:}

Fig. 1. Potential curves of RuC singlet states at FOCI level.

Fig. 2. Potential curves of RuC triplet states at FOCI level.

Fig. 3. Potential curves of RuC quintet states at FOCI level.

Fig. 4. Potential curves of RuC heptet states at FOCI level.

Fig. 5 Electron difference density of ${ }^{1} \Sigma^{+}$state of RuC.

Fig. 6 Electron difference density of ${ }^{3} \Delta(1)$ state of RuC. 